\title{
Uso da cola de cianoacrilato como alternativa em procedimentos cirúrgicos: uma revisão integrativa
}

\author{
Use of cyanoacrylate glue as an alternative in surgical procedures: an integrative review \\ Uso de pegamento de cianoacrilato como alternativa en procedimientos quirúrgicos: una revisión \\ integradora
}

Recebido: 02/02/2021 | Revisado: 06/02/2021 | Aceito: 07/02/2021 | Publicado: 15/02/2021

\author{
José Marcos Valentim Filho \\ ORCID: https://orcid.org/0000-0002-3702-241X \\ Centro Universitário UNIFT, Brasil \\ E-mail: valentimfilho@grupovalentimfilho.com.br \\ Lilamânie Tafnes Santos Pardinho \\ ORCID: https://orcid.org/0000-0001-5934-9811 \\ Centro Universitário UNIFTC, Brasil \\ E-mail: itamarpardinho@hotmail.com \\ Katia de Miranda Avena \\ ORCID: https://orcid.org/0000-0002-2179-3893 \\ Centro Universitário UNIFTC, Brasil \\ E-mail: katiaavena@hotmail.com \\ Victor Araújo Felzemburgh \\ ORCID: https://orcid.org/0000-0002-0880-2687 \\ Centro Universitário UNIFTC, Brasil \\ E-mail: victor.doc@hotmail.com
}

\begin{abstract}
Resumo
Objetivo: Verificar se o uso da cola de cianoacrilato em procedimentos cirúrgicos produz desfechos benéficos para o paciente, no que diz respeito à cicatrização e custo benefício. Métodos: Foi realizada uma revisão integrativa da literatura nas bases de dados PubMed, Bireme/BVS e SciELO, sendo incluídos artigos que se referiam a cianoacrilatos, cirurgias e suturas em seres humanos. Artigos de revisão da literatura, dissertações, capítulos de livros, estudos de caso e editoriais, além dos textos duplicados, foram excluídos. Resultados: Foram encontrados 897 artigos, dos quais 830 foram excluídos após aplicação dos critérios de inclusão e exclusão, e após a leitura dos resumos e textos na íntegra. Assim, 67 artigos foram incluídos nesta revisão. Conclusão: Os estudos demonstraram que as colas de cianoacrilato podem ser utilizadas como alternativa em procedimentos cirúrgicos, principalmente pela facilidade de aplicação, diminuição do risco de contaminação e aumento da eficácia da cicatrização. Apesar de não substituírem as suturas em todos os procedimentos cirúrgicos, as colas de cianoacrilato constituem-se como um método rápido, barato e eficaz, com melhor custo-benefício e tempo de cicatrização, especificamente, nas cirurgias plásticas, oftalmológicas e cardíacas.
\end{abstract}

Palavras-chave: Cianoacrilatos; Procedimentos cirúrgicos operatórios; Cicatrização; Sutura.

\begin{abstract}
Objective: This article aims to verify whether the use of cyanoacrylate glue in surgical procedures produces beneficial outcomes for the patient regarding healing and cost benefit. Methods: An integrative literature review was performed in the PubMed, Bireme/BVS and SciELO databases, including articles referring to cyanoacrylates, surgeries and sutures in humans, published in Portuguese, English and Spanish. Literature review articles, dissertations, book chapters, case studies, and editorials were excluded. Results: A total of 897 articles were found, of which 830 were excluded after applying the inclusion and exclusion criteria, and after reading the abstracts and full texts. Thus, 67 articles were included in this review. Conclusion: Studies have shown that cyanoacrylate glues can be used as an alternative in surgical procedures, mainly due to their ease of application, decreased risk of contamination and increased healing efficiency. Although they do not replace sutures in all surgical procedures, cyanoacrylate glues are a fast, inexpensive and cost-effective method of healing, specifically in plastic, ophthalmic and cardiac surgeries.
\end{abstract}

Keywords: Cyanoacrylates; Surgical procedures operative; Wound healing; Sutures.

\section{Resumen}

Objetivo: Verificar si el uso de pegamento de cianoacrilato en procedimientos quirúrgicos produce resultados beneficiosos para el paciente, en cuanto la cicatrización y coste beneficio. Métodos: Se realizó una revisión integradora de la literatura en las bases de datos PubMed, Bireme / BVS y SciELO, incluyendo artículos referidos a 
cianoacrilatos, cirugías y suturas en humanos. Se excluyeron artículos de revisión de literatura, disertaciones, capítulos de libros, estudios de casos y editoriales, además de los textos duplicados. Resultados: Se encontraron 897 artículos, de los cuales 830 fueron excluidos luego de aplicar los criterios de inclusión y exclusión, y luego de leer los resúmenes y textos completos. Por lo tanto, se incluyeron 67 artículos en esta revisión. Conclusión: Los estudios han demostrado que los adhesivos de cianoacrilato pueden usarse como una alternativa en procedimientos quirúrgicos, principalmente debido a su facilidad de aplicación, menor riesgo de contaminación y mayor eficiencia de curación. Aunque no sustituyen a las suturas en todos los procedimientos quirúrgicos, los adhesivos de cianoacrilato son un método rápido, económico y eficaz, con el mejor costo-beneficio y tiempo de cicatrización, específicamente en cirugías plásticas, oftálmicas y cardíacas.

Palabras clave: Cianoacrilatos; Procedimientos quirúrgicos operativos; Curación; Sutura.

\section{Introdução}

Os procedimentos cirúrgicos, de modo geral, têm um custo elevado tanto para o paciente quanto para o Estado devido, essencialmente, ao alto valor monetário atribuído aos serviços médicos e auxiliares, tempo de uso de centros cirúrgicos, uso de instrumentos de sutura e curativos. No Brasil, no período entre 2008 a 2016, ocorreram mais de 37 milhões de internações cirúrgicas e o custo médio foi em torno de trezentos e noventa dólares (U\$ 390,00) (Covre et al., 2019).

O cianoacrilato é uma substância química, com poder bactericida e bacteriostática, desenvolvida durante a Segunda Guerra Mundial, para colar armas de plástico. Foi sintetizado em 1949, mas apenas na década de 60 passou a ser utilizada em procedimentos cirúrgicos. (D'assumpção, 2008) Seu mecanismo de ação se dá por polimerização. As proteínas orgânicas (catalisadoras) reagem com o cianoacrilato, produzindo calor e uma crosta sobre a superfície onde foi aplicado, mantendo os tecidos interligados. Na presença de água ou sangue, formam longas e fortes cadeias que unem superfícies (Kusabara, Kasahara, 2017). Por estes motivos, alguns procedimentos cirúrgicos vêm substituindo a sutura tradicional pelos adesivos de cianoacrilato (Barbalinardo et al., 1986; De Carvalho, Marchi, 2013; Mobley et al., 2002; Shapiro et al., 2001).

A partir de 2007, observa-se uma maior tendência ao uso de adesivos à base de cianoacrilato em suturas de lesões secas, não infectadas e com bordas acessíveis. Porém, o alto custo de tais adesivos dificulta sua aquisição na maioria dos hospitais brasileiros. Uma alternativa eficaz e com melhor custo-benefício é o uso de colas caseiras à base de cianoacrilato, que tem um valor de mercado muito abaixo dos adesivos utilizados em cirurgias (D'assumpção, 2008; Oliveira et al., 2010).

Estudos sugerem que a utilização de cianoacrilato reduz o tempo operatório, diminui a probabilidade de infecções bacterianas, além de ser um método indolor e de não necessitar de instrumentos especiais de sutura ou anestésicos, reduzindo o custo cirúrgico. Além disso, o uso de cianoacrilato permite a assepsia do local após a cirurgia e dispensa o uso de curativos, favorecendo a cicatrização e aumentando a qualidade de vida dos pacientes (Oliveira et al., 2010).

Frente ao exposto, esta revisão da literatura se propõe a verificar se o uso da cola de cianoacrilato em procedimentos cirúrgicos produz desfechos benéficos para o paciente, no que diz respeito à cicatrização e custo benefício.

\section{Metodologia}

Foi realizada uma revisão da literatura, de natureza quanti-qualitativa (Pereira et al., 2018), seguindo a seguinte sequência lógica: 1) elaboração da pergunta de investigação, com base na estratégia Patient or Problem, Intervention, Control or Comparasion, Outcomes (PICO); 2) identificação das bases de dados para a seleção dos estudos; 3) definição de critérios de inclusão e exclusão; e 4) avaliação da qualidade metodológica dos artigos identificados.

A pesquisa foi registrada no International Prospective Register of Systematic Reviews (PROSPERO), banco de dados internacional de revisões sistemáticas em saúde, que ajuda a evitar a duplicidade de pesquisas, estando cadastrada sob o número CRD42020152494.

Como foram utilizados dados secundários provenientes da literatura, não envolvendo experimentação primária em seres humanos, foi dispensada a submissão ao comitê de ética em pesquisa. 


\section{Estratégia de Busca}

A pergunta de investigação que norteou a estratégia de busca foi: "quais as vantagens, em relação à cicatrização e o custo-benefício, na utilização da cola de cianoacrilato em procedimentos cirúrgicos?”. Assim, no intuito de responder à questão norteadora dessa investigação, foram estabelecidos como desfechos primários: o processo de cicatrização, sendo consideradas as taxas de infecção e de deiscência nos procedimentos pós-cirúrgicos; a relação custo-benefício; e as taxas de complicações do uso de bricolagem (cola de cianoacrilato) em procedimentos cirúrgicos. Como desfechos secundários foram considerados a especialidades em que o procedimento cirúrgico foi realizado, o tipo de material utilizado e a prevalência do uso de cola de cianoacrilato nas cirurgias.

O levantamento dos artigos científicos foi realizado na Biblioteca Nacional de Medicina dos Estados Unidos (PubMed), Biblioteca Virtual em Saúde (BVS/BIREME) e na Scientific Eletronic Library Online (SciELO). Com base nos descritores em Saúde (DECs e MeSH), foram estabelecidas como palavras-chave: cianoacrilato; cirurgia; cicatrização; sutura, e seus correlatos na língua inglesa e espanhola.

O cruzamento entre as palavras-chave foi realizado da seguinte forma: "Cyanoacrylate OR Cyanoacrylates AND Surgery OR Surgeries AND Healing"; "Cyanoacrylates OR Cyanoacrylates AND Surgery OR Surgeries"; "Cyanoacrylate OR Cyanoacrylates AND Healing”; “Cyanoacrylate OR Cyanoacrylates AND Suture”. Essa estratégia de busca foi adaptada para as demais bases de dados. A última atualização ocorreu em 10 de outubro de 2019.

De forma intencional, foram empregados termos mais amplos no intuito de identificar uma maior quantidade de estudos e, assim, minimizar a chance de que algum artigo importante pudesse ser excluído desse levantamento. Além disso, foram verificadas as listas de referência dos estudos primários incluídos na revisão no intuito de identificar possíveis estudos que atendam aos critérios de inclusão, mas que, por algum motivo, não tenham sido identificados através da estratégia de busca definida.

\section{Critérios de Elegibilidade}

Foram incluídos artigos que abordassem o uso de cianoacrilato em procedimentos cirúrgicos. Não foram impostas restrições quanto à data de publicação dos estudos, nem quanto ao idioma das publicações.

Foram excluídos artigos de revisão da literatura, estudos de caso, dissertações, livros, capítulos de livros, editoriais, dentre outros formatos de texto que não tenham passado por rigoroso processo de avaliação por pares, como ocorre com os artigos científicos. Além disso, também foram excluídos os textos encontrados em duplicidade pela indexação em mais de uma base de dados.

\section{Coleta de Dados}

O levantamento dos dados bibliográficos foi realizado de agosto a dezembro de 2019. Após a identificação dos estudos, foi realizada a avaliação crítica destes. Essa etapa consistiu na leitura e análise dos títulos e resumos dos estudos identificados, sendo realizada por dois revisores independentes. Nesse momento, foram excluídos os estudos de revisão e aqueles que não atendiam à proposta desta revisão. Após a triagem inicial, a etapa seguinte consistiu na leitura dos estudos na íntegra, o que possibilitou que alguns textos fossem excluídos por não serem considerados confiáveis (por questões metodológicas, de randomização, conflitos de interesse, dentre outros fatores). Por último, os estudos foram organizados em uma tabela no intuito de apresentar suas principais informações, facilitando a análise descritiva e crítica dos resultados obtidos pelos autores. Os artigos selecionados foram avaliados por dois pesquisadores independentes e, em caso de divergências, o desempate foi feito por um terceiro pesquisador. 


\section{Resultados}

Após o emprego da estratégia de busca, foram identificados 897 resultados. Desse total, 108 artigos foram excluídos por estarem em duplicidade. Após aplicação dos critérios de inclusão e exclusão, foram excluídos outros 184 artigos. Dos 605 artigos restantes, após a leitura na íntegra, outros 546 artigos foram excluídos. Assim, de acordo com os critérios de elegibilidade estabelecidos, 59 artigos compuseram o estudo (Figura 1).

Figura 1. Fluxograma das etapas de identificação, seleção e inclusão dos textos.
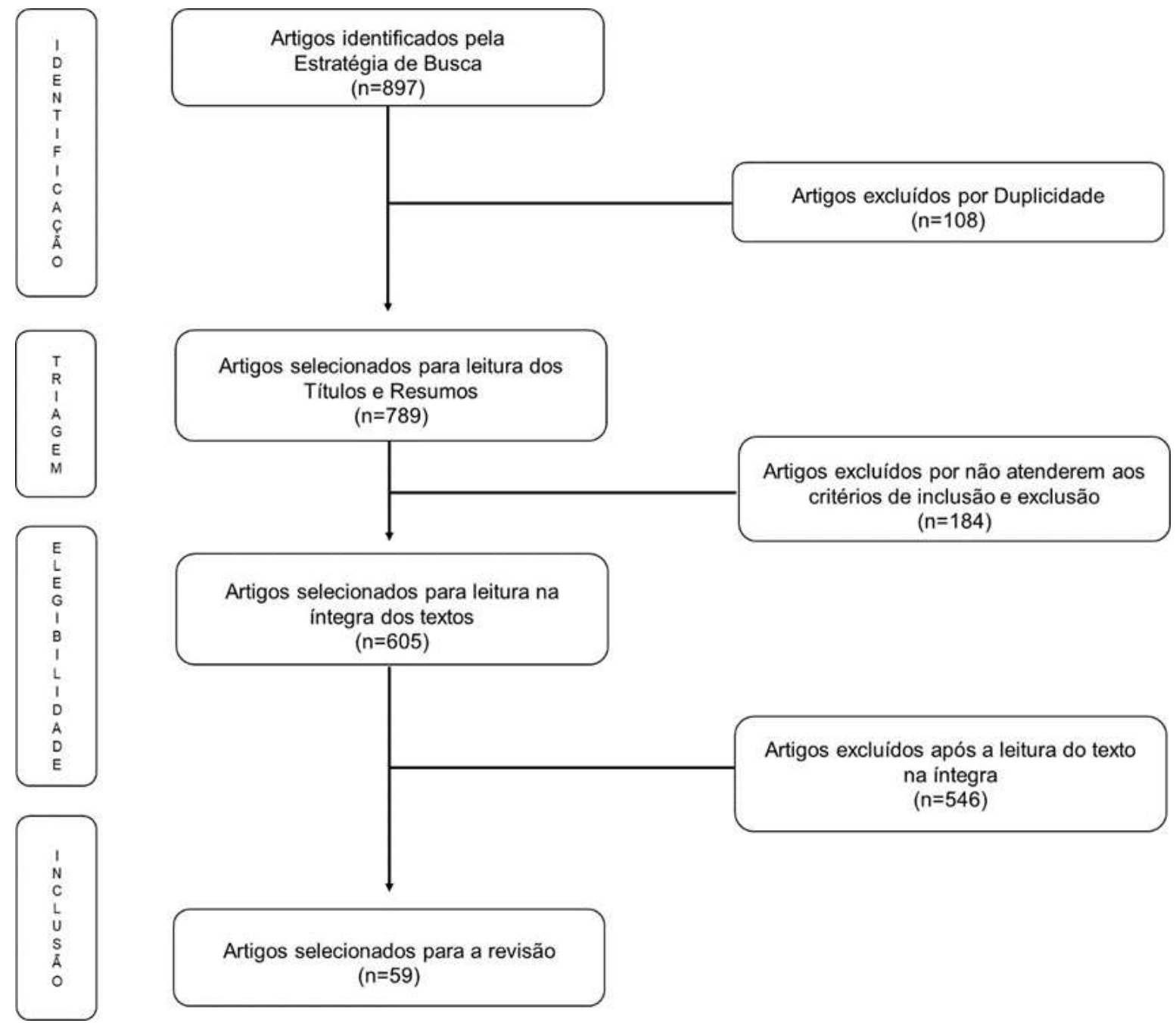

Fonte: Autores.

As características dos estudos incluídos nessa revisão são apresentadas na tabela 1, sendo estes agrupadas por ano de publicação, idioma e área de conhecimento do estudo. Na Tabela 1, observa-se uma concentração na distribuição temporal de publicação dos artigos, havendo destaque para os últimos dez anos. Apenas 10\% dos artigos foi publicado nos primeiros 10 anos (1999-2008). Em relação ao idioma das publicações, 76\% dos artigos foram publicados na língua inglesa, embora as produções sejam oriundas de países distintos. 
Tabela 1. Características dos estudos selecionados quanto ao ano de publicação, idioma e área de conhecimento do estudo $(n=59)$.

\begin{tabular}{lc}
\hline \multicolumn{1}{c}{ CARACTERÍSTICAS } & $\mathbf{n}(\%)$ \\
\hline Ano de Publicação & $04(7)$ \\
$1986-2003$ & $02(3)$ \\
$2004-2008$ & $23(39)$ \\
$2009-2013$ & $30(51)$ \\
$2014-2018$ & \\
Idioma & $45(76)$ \\
Inglês & $09(15)$ \\
Português & $05(9)$ \\
Espanhol & \\
Área de Conhecimento & $07(12)$ \\
Angiologia/Cardiologia & $03(5)$ \\
Buco-maxilo & $14(24)$ \\
Cirurgia Geral & $05(9)$ \\
Cirurgia Plástica & $05(9)$ \\
Dermatologia & $01(2)$ \\
Emergência & $07(12)$ \\
Gastroenterologia & $02(3)$ \\
Ginecologia/Mastologia & $06(10)$ \\
Oftalmologia & $01(2)$ \\
Oncologia & $01(5)$ \\
Ortopedia e Traumatologia & \\
Otorrino & \\
Urologia & $04)$ \\
\hline & \\
\hline
\end{tabular}

Fonte: Autores.

Os estudos incluídos nessa revisão foram desenvolvidos em áreas de conhecimentos, dentro da saúde, distribuídos em quinze especialidades: Angiologia/Cardiologia, Buco-maxilo, Cirurgia Geral, Cirurgia Plástica, Dermatologia, Emergência, Gastroenterologia, Ginecologia/Mastologia, Oftalmologia, Oncologia, Ortopedia, Otorrinolaringologia e Urologia, conforme demonstrado na tabela 1. A distribuição de estudos foi uniforme, com maior destaque para as áreas de Cirurgia Geral (abordada em 24\% dos artigos), e Angiologia/Cardiologia e Gastroenterologia (em 12\%, cada), e Oftalmologia (em 10\%).

Dos estudos analisados, observou-se que $63 \%$ utilizou adesivos de cianoacrilato, um material alternativo para fechamento de feridas, de tecido sintético, de fácil aplicação e alta adesão, enquanto que $37 \%$ utilizaram a cola de cianoacrilato. Com relação ao uso do cianoacrilato como bioadesivo cutâneo, a Tabela 2 apresenta as vantagens e desvantagens levantadas pelos estudos incluídos nessa revisão. 
Tabela 2. Descrição das vantagens e desvantagens do uso de Cianoacrilato.

\begin{tabular}{ll}
\hline VANTAGENS & DESVANTAGENS \\
\hline Aplicação rápida e fácil; & Pouco estudado; \\
Rápido fechamento da pele; & Lacrimejamento; \\
Menor custo; & Odor desagradável; \\
Dispensa curativo e retirada de pontos; & Risco de histotoxidade; \\
Vasta disponibilidade no mercado; & Risco de reações alérgicas; \\
Possibilidade de reesterelizações e de uso repetido; & Uso restrito a feridas lineares, não tensas e \\
& pouco sangrantes. \\
Efeito bactericida, hemostático e selante; & \\
Ausência de marcas de pontos; & \\
Excelentes resultados estéticos; & \\
Grande satisfação dos usuários. & \\
\hline
\end{tabular}

Fonte: Souza \& Briglia (2011).

No tocante ao processo de cicatrização, especificamente às taxas de infecção e de deiscência nos procedimentos póscirúrgicos, os estudos apresentaram bons resultados. Muitos adesivos à base de cianoacrilato foram estudados, incluindo cianoacrilato de isobutil, cianoacrilato de isohexil e cianoacrilato de 2-octilo (OCA). Esses adesivos demonstraram resistência à tração e propriedades bacteriostáticas e hemostáticas.

A prevalência de hematomas e infecções foi relatado em 10\% dos estudos, ou seja, dos 59 artigos selecionados apenas 06 relataram casos de infecção e/ou hematomas no pós-operatório, sendo que as complicações com o uso do cianoacrilato são imediatas, incluindo infecção de feridas e hematomas. Mesmo nos casos onde foi relatado infecção enfatizou-se os efeitos benéficos da diminuição da incidência de hematomas e infecções decorrentes da exposição à urina e fezes em crianças tratadas com o adesivo à prova d'água (Alemayehu et al., 2017).

Com relação às taxas de complicações pelo uso de bricolagem em procedimentos cirúrgicos, foi relatado apenas 01 caso de alergia severa ao uso de adesivo de cianoacrilato (Dermabond) em uma cirurgia mamária. A dermatite de contato com o uso de Dermabond aparece apenas uma vez na literatura, embora a dermatite de contato com cianoacrilato seja mais relatada $(17,5 \%)$ (Perry, Sosin, 2009).

\section{Discussão}

No intuito de verificar se o uso da cola de cianoacrilato em procedimentos cirúrgicos produz desfechos benéficos para o paciente, foram analisados 59 artigos publicados. Considerando as características desses estudos, a concentração das publicações nos últimos dez anos sugere um aumento do interesse na investigação de materiais alternativos, com menor custo, para sutura de procedimentos cirúrgicos. Além disso, a maior frequência das publicações na língua inglesa justifica-se pelo fato de que os periódicos com maior fator de impacto e relevância são publicados nesse idioma.

Por serem áreas mais vascularizadas, os adesivos de cianoacrilato são eficazes para prevenir sangramentos indesejáveis que podem elevar a morbimortalidade pós cirúrgica (Carvalho et al., 2017), sangramentos gastrointestinais e fixação de enxertos ósseos e cutâneos (Vargas et al., 2017). Eles têm sido utilizados na oftalmologia para tratar perfurações da córnea, nas quebras posteriores da retina associadas a descolamentos da retina, para anexar autoenxertos conjuntivais após a excisão do pterígio e para fechar os vazamentos filtrantes da bolha em pacientes com glaucoma (Kusabara, Kasahara, 2017). Observa-se também que, em outras especialidades, como Infectologia, Oncologia e Otorrinolaringologia há poucos estudos 
relevantes, podendo ser compreendido pelo fato de alguns estudos trazerem o cianoacrilato como contraindicado para feridas infectadas e imunocomprometidos (Vargas et al., 2017), fazendo necessário um maior número de ensaios clínicos, prospectivos, randomizados e controlados sobre os seus benefícios, trazendo uma maior adesão a tratamento com risco de imunodepressão, como o antineoplásico (Daeschlein et al., 2014).

Apesar dos adesivos de cianoacrilato já serem usados em cirurgias há mais de 25 anos, nos últimos 10 anos, por serem métodos aprendidos facilmente e com economia do tempo cirúrgico, eles passaram a ser mais vistos (Kim et al., 2018; Silva et al., 2007). Entretanto, esses produtos têm sido subutilizados devido ao alto custo. Em contrapartida, a forma mais economicamente acessível, o 2-etilcianoacrilato (ECA, Aron Alpha, Permabond, cola Krazy, Superbonder®, Epigluß) tem sido pouco estudado como adesivo cutâneo (Souza et al., 2012).

Os artigos que utilizaram cola de cianoacrilato demonstraram que esta é uma forma tão eficaz quanto os adesivos, com um custo-benefício extremamente melhor, o que tem aumentado a frequência do seu uso na prática clínica, em diversas áreas cirúrgicas. (Mikhail et al., 2012; Souza, Briglia, 2011; Souza, Briglia, 2010)

No tratamento de perfurações de córnea, por exemplo, com a utilização da cola de cianoacrilato houve um custo de dezenove reais e oitenta e seis centavos ( $\mathrm{R} \$ 19,86)$, enquanto que com a técnica de sutura de córnea, o mesmo procedimento gastou cento e trinta e oito reais e trinta e oito centavos ( $\mathrm{R} \$ 138,38$ ), o que representou uma diferença significativa de preços entre as duas técnicas (Ambriz-González et al., 2014; Ueda, Ottaiano, 2004).

$\mathrm{O}$ fechamento eficaz da ferida resulta em menor tempo operatório e em menos dor no pós-operatório. Esteticamente, as feridas fechadas com cianoacrilato apresentam um visual muito mais aceitável pelo paciente, principalmente quando comparado às suturas absorvíveis interrompidas (Gorgulu et al., 2016; Perry, Sosin, 2009; Van Haute et al., 2015). No entanto, estudos demonstram diminuição do tempo de fechamento da sutura, quando se utiliza colas (cianoacrilato de N-butil-2) em comparação aos adesivos sintéticos (cianoacrilato de sutura e octil), independentemente do tipo de corte e largura (Krishnamoorthy et al., 2009). Porém, em relação aos adesivos biológicos (fibrina) e sintéticos (cianoacrilato), verificou-se que os adesivos biológicos apresentam menor resistência à tração e menor tempo de polimerização. Além disso, eles têm o potencial de transmissão da doença em comparação com adesivos sintéticos (Koonce et al., 2015).

Com base em estudos nacionais e internacionais, vale ressaltar que, embora haja evidências de boa qualidade do uso da cola de cianoacrilato em cirurgias, esse procedimento, no Brasil, ainda é considerado como experimental visto que ainda não possui registro sanitário.

A questão aqui não é a inexistência de evidências, mas a forma com que uma agência reguladora como a Agência Nacional de Vigilância Sanitária (ANVISA) autoriza a comercialização de produtos para a saúde.

A ação da ANVISA é reativa, não proativa. Assim, um novo registro no Brasil só ocorre se houver um fabricante que o peticione, pague as taxas e junte a documentação necessária. Havendo desinteresse do fabricante da cola de cianoacrilato, cujo exemplo mais conhecido é o, de nome comercial, Superbonder®, em peticionar registro sanitário à ANVISA, nada acontecerá, mesmo que o produto seja eficaz e seguro, com base em evidências suficientemente demonstradas em estudos científicos de boa qualidade metodológica.

Como o uso de produtos sem registro na ANVISA está em desacordo com a legislação sanitária, os estabelecimentos de saúde que usem rotineiramente as colas de cianoacrilato estão sujeitos às penalidades impostas por lei.

Os médicos não devem usá-las de forma rotineira, porém teriam permissão de fazê-lo em basicamente duas situações: sob condições de pesquisa clínica e em situações especiais de ameaça à vida de um doente ou de perda de função de órgão ou sistema (uso compassionado). Em ambos os casos, os procedimentos éticos (ética em pesquisa e ética médica) devem ser cumpridos.

O caso das colas de cianoacrilato pode ser um bom exemplo para o estudo das competências e limitações de órgãos 
reguladores como a ANVISA, que acabam refletindo na relação entre médicos e seus pacientes e entre médicos e seus conselhos profissionais.

\section{Conclusão}

Os estudos analisados apontam que as colas de cianoacrilato, de modo geral, podem ser utilizadas como alternativa em procedimentos cirúrgicos, principalmente pela facilidade de aplicação, diminuição do risco de contaminação e aumento da eficácia da cicatrização, devido a menor exposição do tecido submetido à cirurgia. Entretanto, as colas de cianoacrilato não substituem as suturas em todos os tipos de procedimentos, mas se demonstraram eficazes em ferimentos cutâneos, tais como: incisões ou lacerações, possibilitando aposição das bordas do ferimento, sem necessidade de curativos oclusivos, devido a rápida aplicação, resistência à infecção, propriedades hemostáticas e a não necessidade de posterior remoção, diferentemente das suturas não absorvíveis convencionais.

Com relação ao custo-benefício, a cola de cianoacrilato pode ser considerada como um excelente tratamento alternativo, sem necessidade de internação cirúrgica, em nível ambulatorial, sem sedação ou anestesia, sugerindo a possibilidade de redução dos custos com internação hospitalar.

Estudos sugerem também que, analisando o estado de saúde mental, com a redução do tempo médio de internação e cirurgia, principalmente crianças e idosos, este procedimento alivia o nível de estresse ou ansiedade psicológica.

Apesar de estudos trazerem a deiscência como uma preocupação com o uso dos cianoacrilatos, especialmente em feridas com bordas irregulares ou traumatizadas, em lesões localizadas nas extremidades ou em zonas de pele tensa, sem suturas relaxadoras profundas, os artigos revisados demonstraram que o uso de cola de cianoacrilato constitui-se de método rápido, barato e eficaz, com melhor custo-benefício, tempo de cicatrização e estética nas cirurgias de modo geral, especificamente, nas de cirurgias plásticas, oftalmológicas e cardíacas, onde os resultados dos estudos são mais evidentes.

Por fim, apesar do quantitativo expressivo de estudos publicados sobre o tema, verifica-se que ainda são necessários ensaios clínicos randomizados que comparem a sutura tradicional e a utilização da cola de cianoacrilato em procedimentos cirúrgicos para verificar seu efeito no processo de cicatrização e nas taxas de infecção, de deiscência e de complicações.

\section{Referências}

Alemayehu, H., Sharp, N. E., Gonzalez, K., Poola, A. S., Snyder, C. L., \& Peter, S. D. S. (2017). The role of 2-octyl cyanoacrylate in prevention of penile adhesions after circumcision: A prospective, randomized trial. Journal of pediatric surgery, 52(12), 1886-1890.

Ambriz-Gonzalez, G., Aguirre-Ramirez, P., García-de León, J. M., León-Frutos, F. J., Montero-Cruz, S. A., Trujillo, X., ... \& Gonzalez-Ojeda, A. (2014). 2octyl cyanoacrylate versus reintervention for closure of urethrocutaneous fistulae after urethroplasty for hypospadias: a randomized controlled trial. BMC urology, 14(1), 1-6.

Barbalinardo, R. J., Citrin, P., Franco, C. D., \& Hobson II, R. W. (1986). A comparison of isobutyl 2-cyanoacrylate glue, fibrin adhesive, and oxidized regenerated cellulose for control of needle hole bleeding from polytetrafluoroethylene vascular prostheses. Journal of vascular surgery, 4(3), $220-223$.

Carvalho, M. V. H. D., Marchi, E., \& Lourenço, E. A. (2017). Comparison of arterial repair through the suture, suture with fibrin or cyanoacrylate adhesive in ex-vivo porcine aortic segment. Brazilian journal of cardiovascular surgery, 32(6), 487-491.

Covre, E. R., Melo, W. A. D., Tostes, M. F. D. P., \& Fernandes, C. A. M. (2019). Permanência, custo e mortalidade relacionados às internações cirúrgicas pelo Sistema Único de Saúde. Revista Latino-Americana de Enfermagem, 27.

Daeschlein, G., Napp, M., Assadian, O., Bluhm, J., Krueger, C., von Podewils, S., Kramer, A. (2014). Influence of preoperative skin sealing with cyanoacrylate on microbial contamination of surgical wounds following trauma surgery: a prospective, blinded, controlled observational study. International Journal of Infectious Diseases, 29, 274-278.

D'Assumpção, E. A. (2008). Cola de cianoacrilato de baixo custo em cirurgia plástica. Rev Soc Bras Cir Plast, 23(1), $22-5$.

de Carvalho, M. V. H., \& Marchi, E. (2013). Mecanismo de ação dos agentes hemostáticos locais e adesivos tissulares. Rev Med Minas Gerais, 23(4), 504509.

Gorgulu, T., Olgun, A., Torun, M., \& Kargi, E. (2016). A fast, easy circumcision procedure combining a CO2 laser and cyanoacrylate adhesive: a nonrandomized comparative trial. International braz j urol, 42(1), 113-117. 
Research, Society and Development, v. 10, n. 2, e28310212592, 2021

(CC BY 4.0) | ISSN 2525-3409 | DOI: http://dx.doi.org/10.33448/rsd-v10i2.12592

Kim, H., Kim, W., Kang, G. H., Jang, Y. S., Choi, H. Y., Kim, J. G., \& Kim, M. (2018). Comparison of Leukosan SkinLink with surgical suture for traumatic laceration repair: A randomized controlled trial. Medicine, $97(25)$.

Koonce, S. L., Eck, D. L., Shaddix, K. K., \& Perdikis, G. (2015). A prospective randomized controlled trial comparing N-butyl-2 cyanoacrylate (Histoacryl), octyl cyanoacrylate (Dermabond), and subcuticular suture for closure of surgical incisions. Annals of plastic surgery, 74(1), 107-110.

Krishnamoorthy, B., Najam, O., Khan, U. A., Waterworth, P., Fildes, J. E., \& Yonan, N. (2009). Randomized prospective study comparing conventional subcuticular skin closure with Dermabond skin glue after saphenous vein harvesting. The Annals of thoracic surgery, 88(5), 1445-1449.

Kusabara, A. A., \& Kasahara, N. (2017). Managing glaucoma drainage device tube leak with cyanoacrylate. Acta ophthalmologica, 95(7), e662-e662.

Mikhail, A. E., Luquero, A. P., Pascual, J. F. R., \& Gil, J. L. S. (2012). Fijación del material protésico en la hernioplastia inguinal abierta: sutura Vs. cola sintética. Cirugía Española, 90(7), 446-452.

Mobley, S. R., Hilinski, J., \& Toriumi, D. M. (2002). Surgical tissue adhesives. Facial Plastic Surgery Clinics, 10(2), 147-154.

Oliveira, C. L. D., Santos, C. H. M. D., Bezerra, F. M. M., Bezerra, M. M., \& Rodrigues, L. D. L. (2010). Utilização de adesi vos de cianoacrilatos em suturas de pele. Revista Brasileira de Cirurgia Plástica, 25(3), 573-576.

Pereira, A. S., Shitsuka, D. M., Parreira, F. J., \& Shitsuka, R. (2018). Metodologia da pesquisa científica. UFSM. Recuperado de: https://repositorio.ufsm.br/bitstream/handle/1/15824/Lic_Computacao_Metodologia-Pesquisa-Cientifica.pdf?sequence=1.

Perry, A. W., \& Sosin, M. (2009). Severe allergic reaction to Dermabond. Aesthetic surgery journal, 29(4), 314-316.

Shapiro, A. J., Dinsmore, R. C., \& North Jr, J. H. (2001). Tensile strength of would closure with cyanoacrylate glue. The American Surgeon, 67(11), 1113.

Silva, L. S., Neto, J. B. F., \& Santos, A. L. Q. (2007). Utilização de adesivos teciduais em cirurgias-Revisão. Bioscience Journal, 23(4).

Souza, S. C. D., \& Briglia, C. H. (2011). Estudo comparativo entre etilcianoacrilato e sutura intradérmica no fechamento de excisões cutâneas. Revista Brasileira de Cirurgia Plástica, 26(4), 566-572.

Souza, S. C. D., \& Briglia, C. H. (2010). Reparo de fissura de lóbulo auricular usando cola cirúrgica de menor custo. Revista Brasileira de Cirurgia Plástica, 25(4), 715-718.

Ueda, E. L., \& Ottaiano, J. A. A. (2004). Comparação dos gastos com material para reparação de perfurações de córnea com cola de cianoacrilato e sutura de córnea. Arquivos Brasileiros de Oftalmologia, 67(1), 97-101.

Van Haute, C., Tailly, T., Klockaerts, K., \& Ringoir, Y. (2015). Sutureless circumcision using 2-Octyl cyanoacrylate results in more rapid and less painful procedures with excellent cosmetic satisfaction. Journal of pediatric urology, 11(3), 147-e1.

Vargas, A., Foncea, C., \& Campolo, A. (2017). Adhesivos de cianoacrilato en cirugía oral y maxilofacial. Revista clínica de periodoncia, implantologia y rehabilitación oral, 10(2), 107-110. 